\title{
Wideband Dual-Layer Patch Antenna Fed by a Modified L-Strip
}

\author{
Zhongbao Wang*, Shaojun Fang, and Shiqiang Fu \\ School of Information Science and Technology, Dalian Maritime University, Liaoning116026, China \\ *e-mail:wangzb@dlmu.edu.cn
}

\begin{abstract}
A wideband patch antenna is proposed. By using the dual-layer patch configuration and modified L-strip feed technique, the impedance bandwidth of the patch antenna for $V S W R<1.2$ is broadened to $22 \%$ with respect to $900 \mathrm{MHz}$, which is superior to the other available patch antennas. In addition, by means of the modified L-shaped strip, the high cross polarization levels of the proposed antenna are suppressed effectively. Simulation results are compared with the measurements, and a good agreement is obtained. The measurements show that the antenna has a low crosspolarization level (<-20dB) and an acceptable gain level ( 9dBi) across the operating bandwidth. At last, a parametric study and a design guideline of the proposed antenna are also presented to facilitate the design and optimization processes for engineers.
\end{abstract}

Index Terms-good impedance matching, cross-polarization suppression, wideband, modified L-strip, dual-layer patch antenna.

\section{INTRODUCTION}

Currently, microstrip patch antennas are widely used due to their low cost, light weight, and ease of manufacture. However, a single patch antenna has a narrow impedance bandwidth. Some excellent techniques [1]-[7] have been proposed to broaden the impedance bandwidth, including the use of thick and low-permittivity dielectric substrates, different shape patches [1], [2] and ground [3], [4], addition of parasitic patches [5], [6]. For examples, the two E-shaped stacked patch antenna achieves an impedance bandwidth of 54\% [5] for VSWR $<2$ and the impedance bandwidth is further increased to $59.7 \%$ [6] by using a U-slotted patch instead of the fed E-shaped patch. More recent research on probe-fed stacked patch for ultra-wideband (UWB) applications has resulted in $102.36 \%$ bandwidth [7]. In light of the foregoing survey, using the VSWR $<1.2$ requirement, the impedance bandwidth of the available patch antennas is still narrow (less than 10\%). As we known, the VSWR of the antennas for DAB (Digital Audio Broadcasting) and DVB (Digital Video Broadcasting) transmission system is needed to be less than 1.2. So the colossal growth of DAB and DVB necessitates the development in the VSWR $<1.2$ bandwidth broadening techniques.

Another issue is that the foregoing techniques [1]-[7] have the disadvantage of high crosspolarization levels. In particular, the beam widths with the cross-polarization levels $20 \mathrm{~dB}$ lower than co-polarization levels are only few degrees near the boresight direction in H-plane. This is evidently a 
limitation to the applications, such as adaptive antenna arrays for cellular or satellite communications, where wide coverage is usually required. In view of this, using differential feeds [8], a half wavelength strip [9], or a meandered strip [10], the cross-polarization can be suppressed effectively. However, the method of differential feeds [8] needs a $180^{\circ}$ wideband feeding network that increases the complexity of the antenna configuration. For [9], the cross-polarization levels would be increased dramatically if using a higher patch height $\left(>0.06 \lambda_{0}\right)$. In addition, the meandering strip [10] is not easily manufactured, especially when the substrate is used.

To solve these problems, a novel wideband patch antenna is proposed in this paper. By using the dual-layer patch configuration and modified L-strip feed technique, the impedance bandwidth of the patch antenna for VSWR $<1.2$ is broadened to $22 \%$ with respect to $900 \mathrm{MHz}$. Furthermore, by means of the modified L-strip, the high cross polarization levels of the proposed antenna are suppressed effectively. The antenna has a cross-polarization level of less than $-20 \mathrm{~dB}$ and a gain level of about $9 \mathrm{dBi}$ across the operating bandwidth. In the next section, the antenna structure is presented, followed by experimental results and analysis.

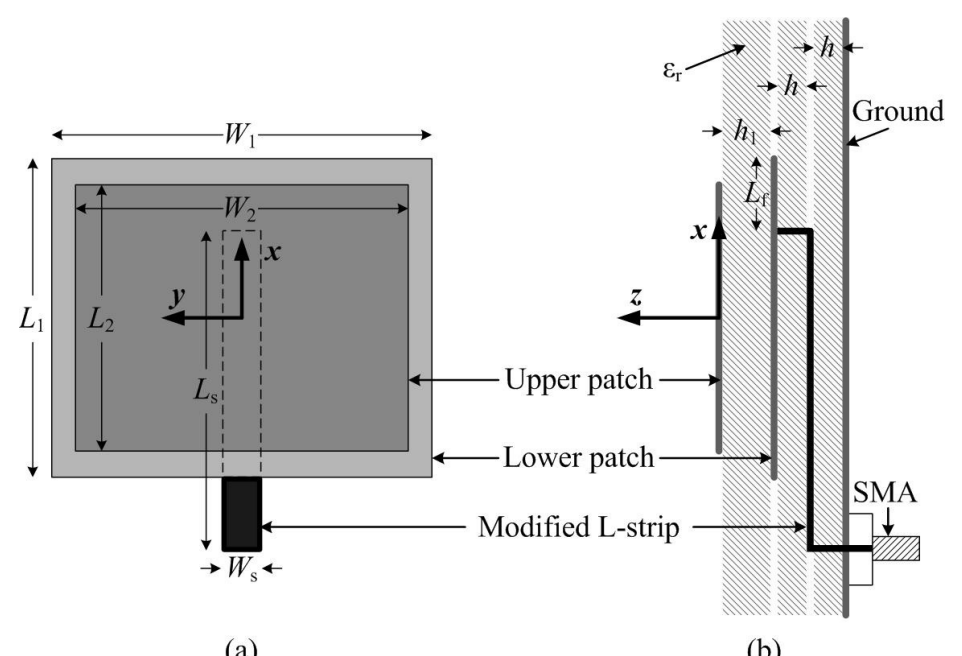

(a)

(b)

Fig. 1. Geometry of the proposed antenna.

\section{ANTENNA STRUCTURE}

The geometry of the stacked patch antenna is shown in Fig. 1. The centre frequency of the proposed antenna is chosen at $f_{0}=900 \mathrm{MHz}\left(\lambda_{0}=333 \mathrm{~mm}\right)$, and the antenna dimensions are determined after a detailed parametric study of the performance of the antenna. The feed strip, lower and upper patches are made of the $0.1 \mathrm{~mm}$ thick copper foil, which are supported by foam layers. The relative dielectric constant of the foam is 1.05 . The lower and upper patches are rectangle in shape and have dimensions $L_{1} \times W_{1}=134 \times 160 \mathrm{~mm}^{2}\left(0.40 \lambda_{0} \times 0.48 \lambda_{0}\right)$ and $L_{2} \times W_{2}=113 \times 140 \mathrm{~mm}^{2}\left(0.34 \lambda_{0} \times 0.42 \lambda_{0}\right)$, respectively. The antenna has a ground plane with dimensions $300 \mathrm{~mm} \times 350 \mathrm{~mm}\left(0.9 \lambda_{0} \times 1.05 \lambda_{0}\right)$ and the patch position is placed at the centre of the ground plane. The spacing between the lower patch and the ground is chosen as $18 \mathrm{~mm}\left(0.05 \lambda_{0}\right)$. To broaden the impedance bandwidth, the height $h_{1}$ between the upper 
patch and the lower patch is optimized to $24.5 \mathrm{~mm}\left(0.07 \lambda_{0}\right)$. A narrow copper strip of dimensions $W_{\mathrm{s}} \times L_{\mathrm{s}}=14.5 \times 133 \mathrm{~mm}^{2}$ is horizontally placed between the lower patch and the ground both at the distance of $9 \mathrm{~mm}$ and is symmetrically located along the midline of the patches. A $50 \Omega$ SMA connector excites one end of the strip with the other end vertically extended to the lower patch. By properly selecting the distance between the vertical strip and the lower patch edge $\left(L_{\mathrm{f}}=37 \mathrm{~mm}\right)$, one can control impedance matching condition between the probe-fed strip and the lower patch.

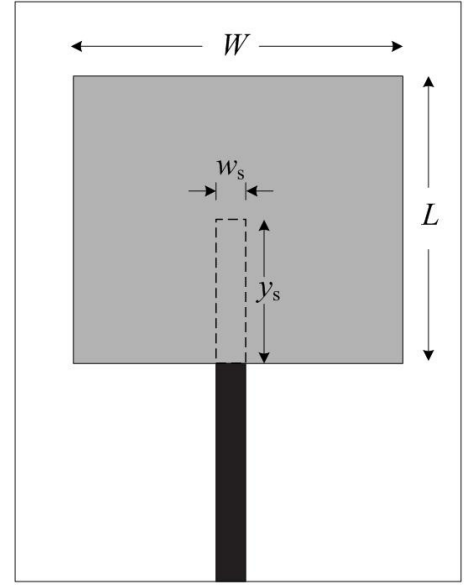

(a)

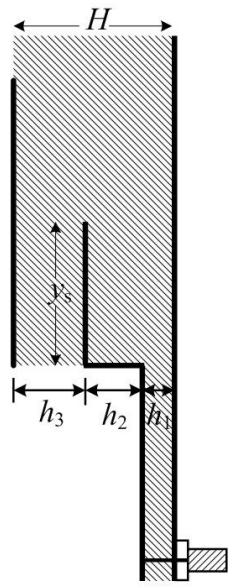

(b)

Fig. 2. L-strip proximity fed rectangular patch antenna presented in [11].

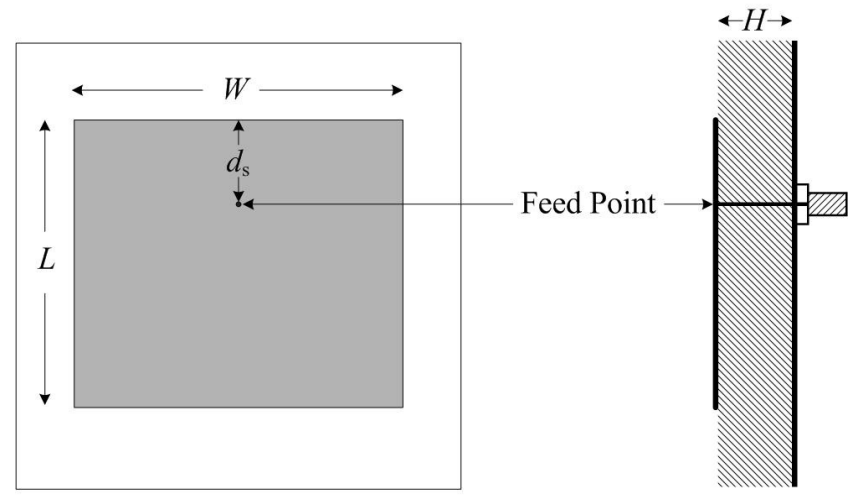

(a)

(b)

Fig. 3. Probe-fed rectangular patch antenna presented in [12].

The feed structure of the proposed antenna is a little similar to the L-strip proximity feed shown in Fig. 2. It is well known that L-strip proximity fed patch antenna is a popular wideband antenna, and impedance matching can be obtained by adjusting the length of the vertical and horizontal portion of the L-strip $\left(h_{2}\right.$ and $\left.y_{s}\right)$. However, the effects of $h_{2}$ and $y_{s}$ on input resistance and reactance are dependent, which result in a complex design process. Fig. 3 gives a probe-fed patch antenna. The probe-fed technique is early proposed and widely used. One advantage of the probe-fed patch antenna 
[12] is that the input resistance is only determined by the feed point $\left(d_{s}\right)$. Base on the L-strip proximity feed and probe-fed techniques, a novel feed structure is introduced and named modified L-strip feed in this paper. The modified L-strip feed structure can easily determine input resistance and reactance, which is certified by parametric studies in the section IV. Furthermore, the modified L-strip feed structure with two vertical segments is similar to the differential feed scheme [9]. Therefore, the high cross polarization levels of the proposed antenna can be suppressed effectively. However, the present scheme differs from those of [9], in which a broadband impedance matching network is inserted by means of the electromagnetic coupling between the modified L-strip and the lower patch.

\section{EXPERIMENTAL RESULTS AND ANALYSIS}

The VSWR of the fabricated prototype were measured by an HP8753D vector network analyzer and the far-field performances were obtained using the SATIMO measurement system in an anechoic chamber. In addition, the commercial software called HFSS (High Frequency Structure Simulator) was also used to simulate the antenna performance.

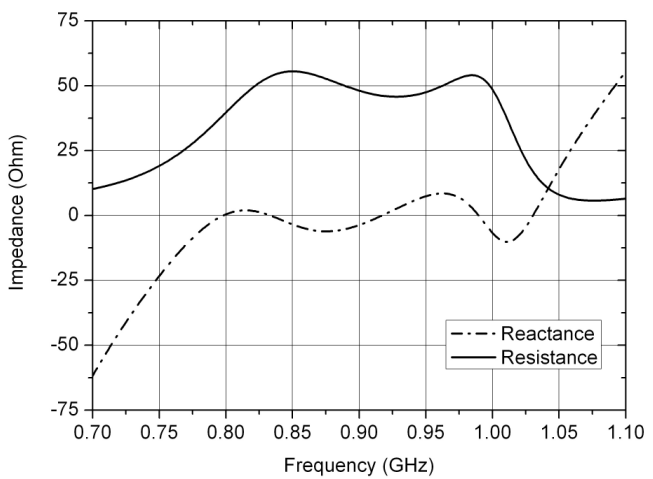

Fig. 4. input impedance of the proposed antenna

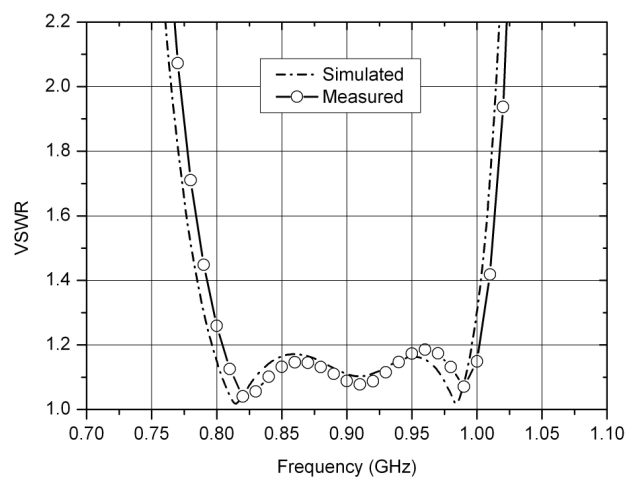

Fig. 5. Comparison of the simulated and measured VSWR.

Fig. 4 gives the simulated input impedance curve. It is clearly observed that the input resistance varies around the $50 \Omega$ and the input reactance keeps small within a broad frequency range to achieve a good impedance matching. The large reactance usually resulted from the feed probe can effectively be cancelled out by the strong electromagnetic coupling, such as coupling between the feed strip and the lower patch. The $50 \Omega$ resistance can be obtained by properly selecting the position of the feed point at the lower patch. So good impedance matching can be obtained by adjusting the size of the antenna $\left(L_{\mathrm{f}}, L_{\mathrm{s}}, W_{\mathrm{s}}\right.$, and $\left.h_{1}\right)$.

A comparison between the simulated and measured VSWR shows good agreement, as displayed in Figure 5. The VSWR curve of the proposed antenna is similar to that of a third-order chebyshev filter, which implies that there are three resonance frequencies of the proposed antenna well excited around $900 \mathrm{MHz}$. Consequently, the impedance bandwidth of the proposed antenna can reach up to $22 \%$ from $804 \mathrm{MHz}$ to $1002 \mathrm{MHz}$ for VSWR<1.2, which attributes to the dual-layer patch configuration and 
modified L-strip feed technique. A comparison of the simulated and measured gain against the frequency is shown in Fig. 6. The antenna has a gain level of about $9 \mathrm{dBi}$ across the operating bandwidth.

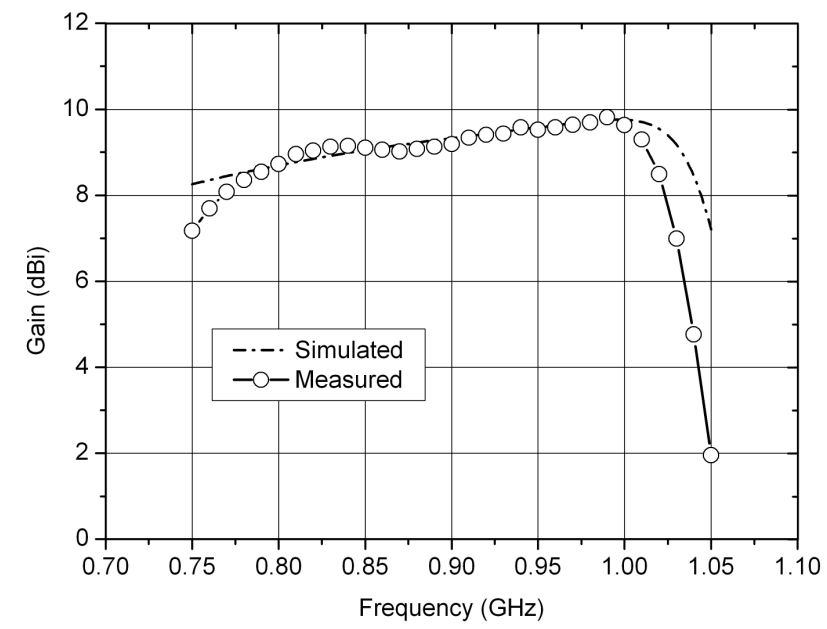

Fig. 6. Comparison of the simulated and measured antenna gain.

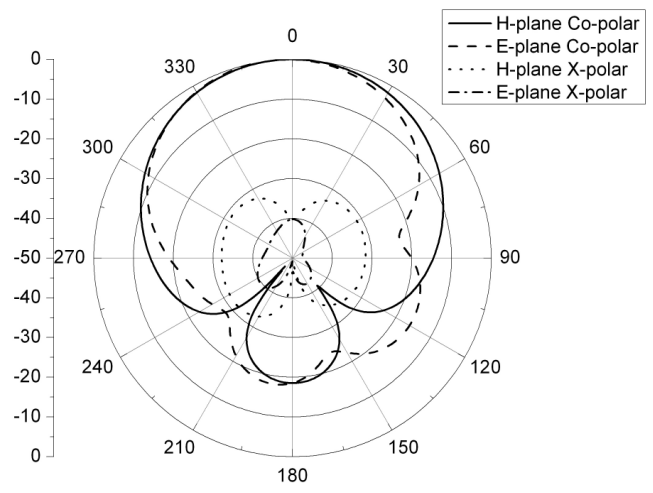

(a)

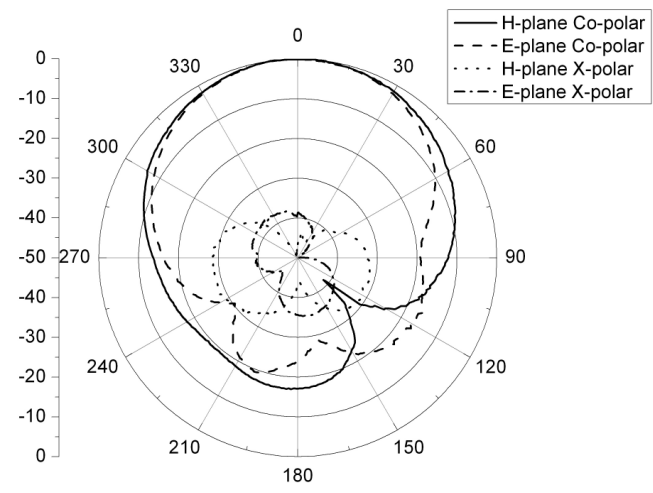

(b)

Fig. 7. Radiation patterns of the proposed antenna at 804MHz: (a) simulated and (b) measured.

Fig. 7, 8 and 9 give the simulated and measured radiation patterns of the proposed antenna in Eplane (x-z) and H-plane (y-z) at 804, 900 and $1002 \mathrm{MHz}$, respectively. It is observed that both of the simulated and measured cross-polarization levels are $20 \mathrm{~dB}$ or more below the maximum copolarization levels within the operating frequencies. The radiation performances of the probe-fed patch antennas is investigated experimentally by Z. N. Chen and M. Y. W. Chia [13]. Their studies clearly show that the degradation of the radiation performances of the broadband probe-fed patch antennas stems significantly from the excitation of higher order modes rather than the probe-radiation. The higher order modes distort the distributions of the induced electric currents, especially near the feed points at the patch. Typically, on boresight, the cross-polarization level remains fairly low, 
however off-boresight angles in the H-plane, the cross-polarization levels can be increased dramatically. In the E-plane, the effect of the higher order modes is to distort the co-polarized pattern, usually causing an undesirable null on one side. In this study, by the introduction of a modified Lstrip, the effect of the higher order modes can be significantly reduced. This is because the two vertical segments at the front and back end of strip with the currents of a $180^{\circ}$ phase shift act as the dual-probe feeding structure [9]. The resultant cross-polarization levels in H-planes come down.

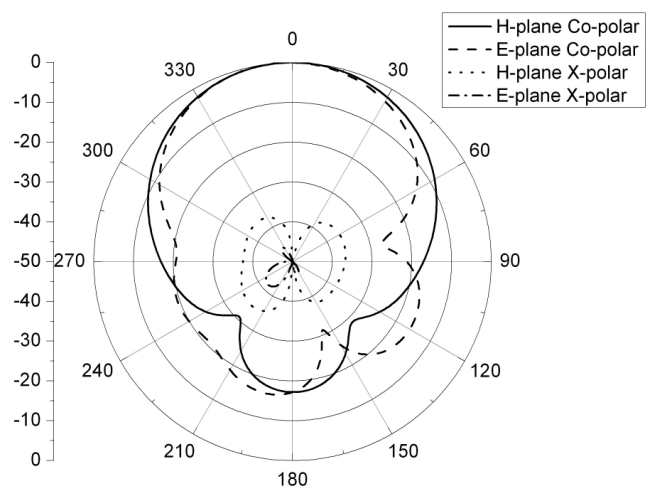

(a)

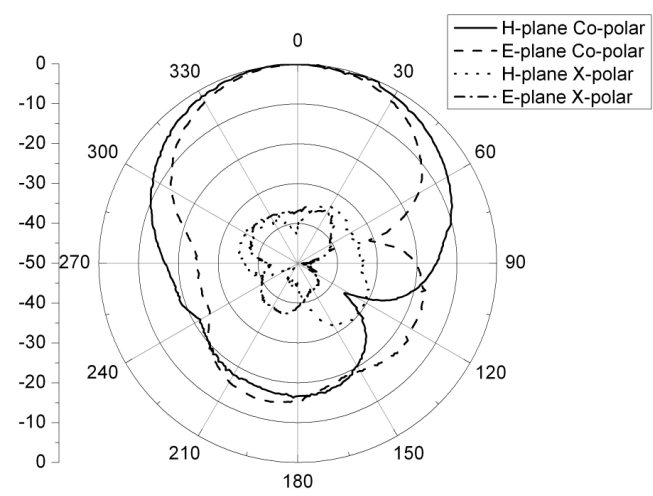

(b)

Fig. 8. Radiation patterns of the proposed antenna at 900MHz: (a) simulated and (b) measured.

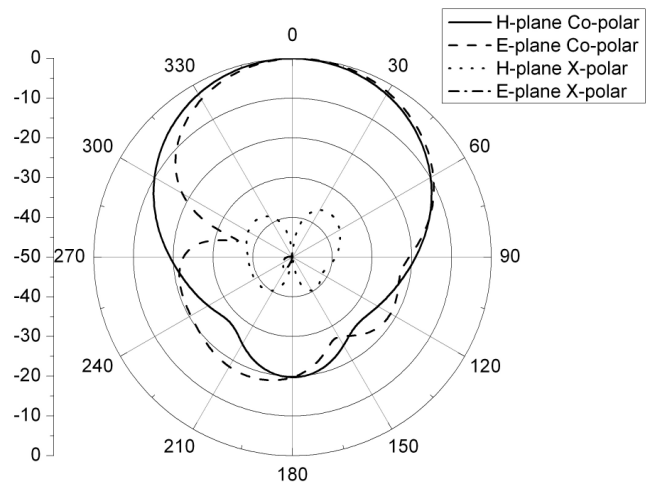

(a)

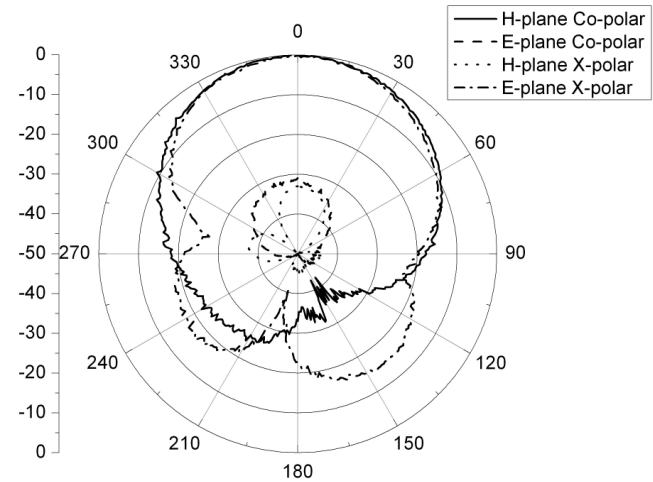

(b)

Fig. 9. Radiation patterns of the proposed antenna at 1002MHz: (a) simulated and (b) measured.

TABLE I

HALF POWER BEAMWITHS

\begin{tabular}{|c|c|c|c|c|}
\hline \multirow{2}{*}{$\begin{array}{c}\text { Frequency } \\
(\mathrm{MHz})\end{array}$} & \multicolumn{4}{|c|}{ Half power beamwidths } \\
\cline { 2 - 5 } & \multicolumn{2}{|c|}{ E-plane } & \multicolumn{2}{c|}{ H-plane } \\
\cline { 2 - 5 } & Simulated & Measured & Simulated & Measured \\
\hline 804 & $65^{\circ}$ & $67^{\circ}$ & $76^{\circ}$ & $78^{\circ}$ \\
\hline 900 & $60^{\circ}$ & $58^{\circ}$ & $69^{\circ}$ & $75^{\circ}$ \\
\hline 1002 & $62^{\circ}$ & $58^{\circ}$ & $64^{\circ}$ & $62^{\circ}$ \\
\hline
\end{tabular}


Simulated and measured results of the half power beamwidth extracted from the radiation patterns are summarized in Table I. The measured half power beamwidths are $58^{\circ}$ in the E-plane and $75^{\circ}$ in the H-plane at the center frequency $(900 \mathrm{MHz})$, which is in good agreement with the simulated half power beamwidths of $60^{\circ}$ in the E-plane and $69^{\circ}$ in the H-plane. Results in Table I show that the half power beamwidths are stable in both E- and H-planes within the operating bandwidth.

At last, the back-lobe radiation levels of the proposed antenna are less than $-16 \mathrm{~dB}$ within the operating bandwidth, which are somewhat low mainly due to the use of the finite-size ground plane in the tests.

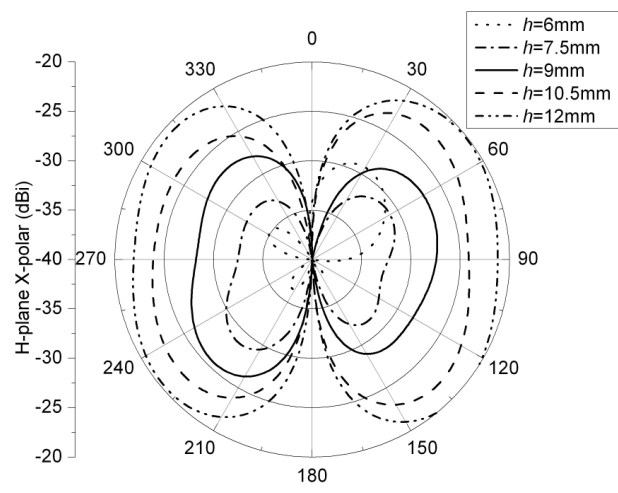

(a)

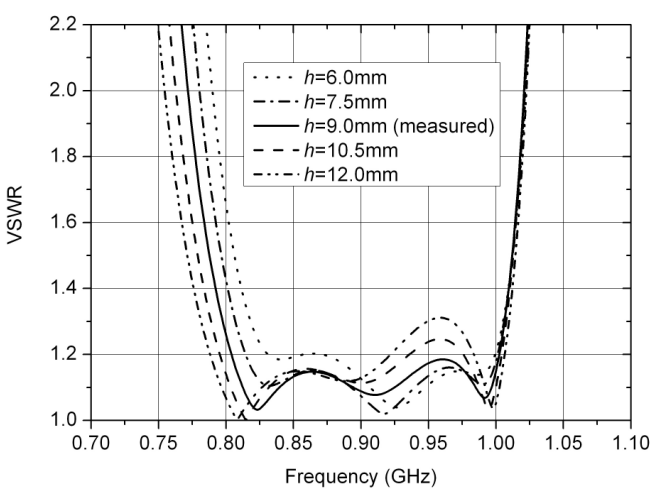

(b)

Fig. 10 Effect of the height of the lower patch on the antenna performance. (a) H-plane x-polar at 900MHz. (b) VSWR.

\section{PARAMETRIC STUDIES AND DESIGN GUIDELINE}

Parametric studies are conducted to provide more detailed information about the antenna design and optimization. The parametric study is carried out by simulation because good agreement between the simulation and measurement has been observed. The parameters under study include the height of the patches, the distance between the vertical segment of the modified L-strip and the edge of the lower patch, the width of the modified L-strip, and the length of horizontal segment of the modified L-strip. Since the effects of some parameters (such as the size of the patches) and the gain performance of the dual-layer patch antenna, have been well known, the study of these parameters and performances is excluded in this paper. To better understand the influence of the parameters on the performance of the antenna, only one parameter at a time will be varied, while others are kept unchanged unless especially indicated.

\section{A. Height of the lower patches $(2 * h)$}

Fig. 10 shows the effect of the height of the lower patch on the H-plane cross-polarization and VSWR of the antenna. It is found that increasing the height $h$ from $9 \mathrm{~mm}$ to $12 \mathrm{~mm}$ causes higher cross-polarization levels and a narrow impedance bandwidth due to the larger inductance shown in Fig. 11. The decreasing of $h$ drops down the cross-polarization levels and achieves better impedance matching. However, over decreasing of $h$ (such as $h=6 \mathrm{~mm}$ ) will degrade the impedance bandwidths. 
In practical design, the height of the lower patch can be optimized for specific design requirement.

\section{B. Height of the upper patches $\left(h_{1}\right)$}

As illustrated in Fig. 12, the input resistances of the antenna decrease at lower band ranging from $800 \mathrm{MHz}$ to $900 \mathrm{MHz}$ when changing the height $\mathrm{h}_{1}$ from $21.5 \mathrm{~mm}$ to $27.5 \mathrm{~mm}$, but increase at upper band ranging from $900 \mathrm{MHz}$ to $1000 \mathrm{MHz}$. Therefore, a flatter input resistance curve can be obtained by tuning the height $h_{l}$. Similar to $h, h_{l}$ has a greater effect on the impedance bandwidths, while the cross-polarization levels of the antenna is modestly affected if $h_{l}$ is kept within $21.5-27.5 \mathrm{~mm}$ as shown in Fig. 13.

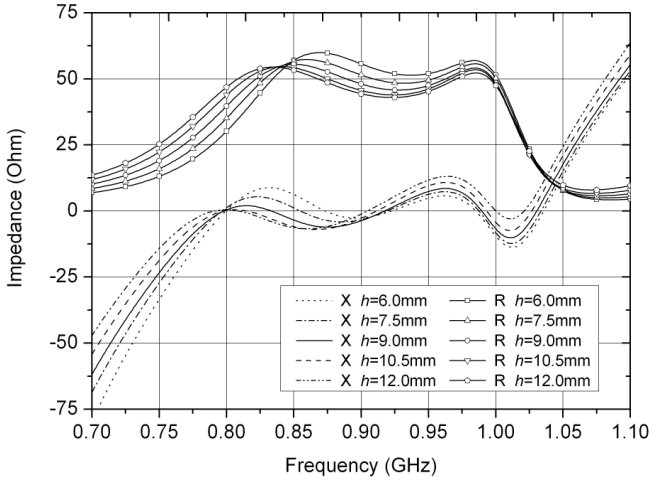

Fig. 11 Effect of the $h$ on the input impedance.

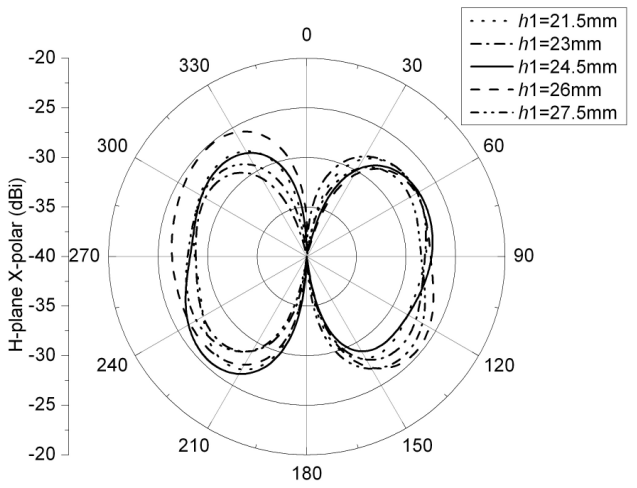

(a)

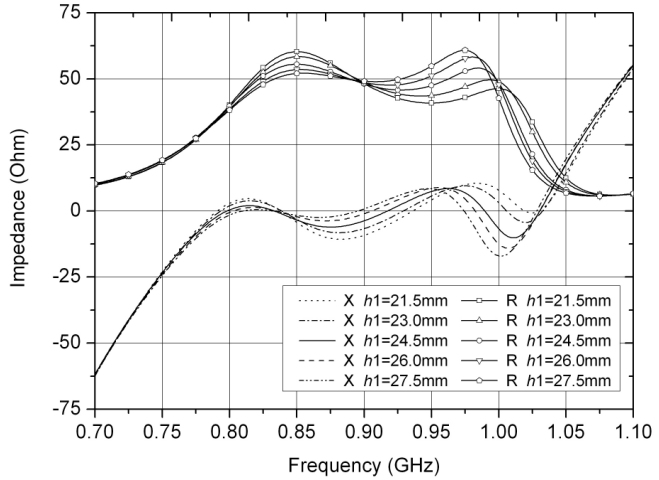

Fig. 12 Effect of the $h_{1}$ on the input impedance.

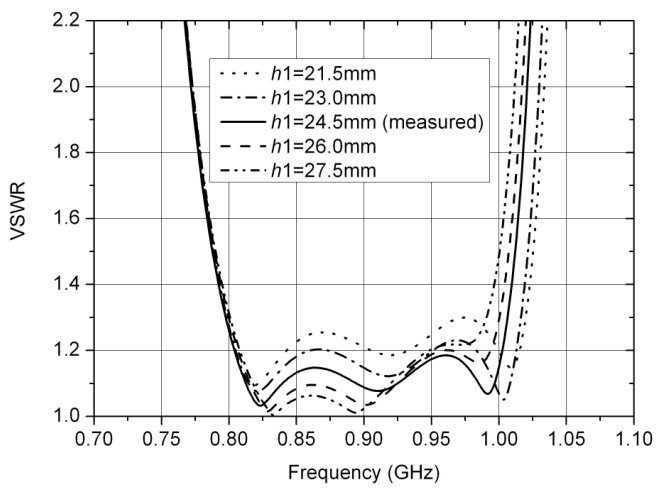

(b)

Fig. 13 Effect of the height of the upper patch on the antenna performance. (a) H-plane x-polar at 900MHz. (b) VSWR.

\section{Distance between Vertical Segment of Modified L-Strip and Edge of Lower Patch $\left(L_{f}\right)$}

Fig. 14 gives the effect of $L_{f}$ on the H-plane cross-polarization and input impedance of the antenna. It is found that the cross-polarization level diminishes as the feed point close to the edge of the lower patch and the input resistance increases, this variation of which is similar to that of the probe-fed patch antenna [12]. The maximum input resistance can be achieved by locating the feed point at the edge of the lower patch. The magnitude of input resistance can vary from a few Ohms to about one 
hundred Ohms. Compare to the probe-fed single-layer rectangular patch antenna (the maximum input resistance is about $140 \mathrm{Ohm}$ for the first resonant mode) [12], the maximum input resistance of the antenna is less. This means that the feed point of the dual-layer patch antenna closer than that of probe-fed single-layer patch antenna to the edge of the patch under the condition of $50 \Omega$ impedance matching. Therefore, the dual-layer patch configuration lowers the cross-polarization level in some degree.

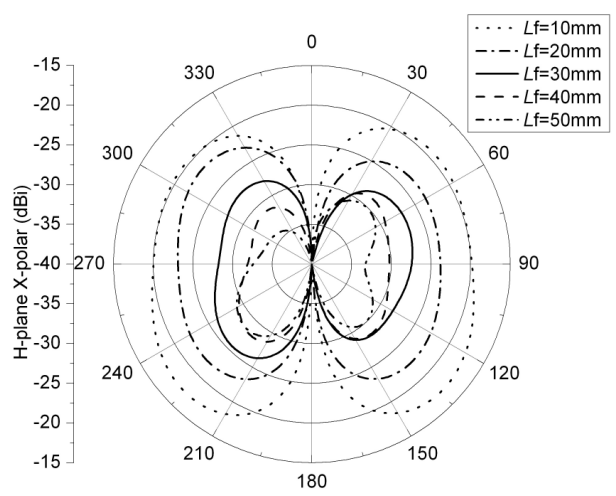

(a)

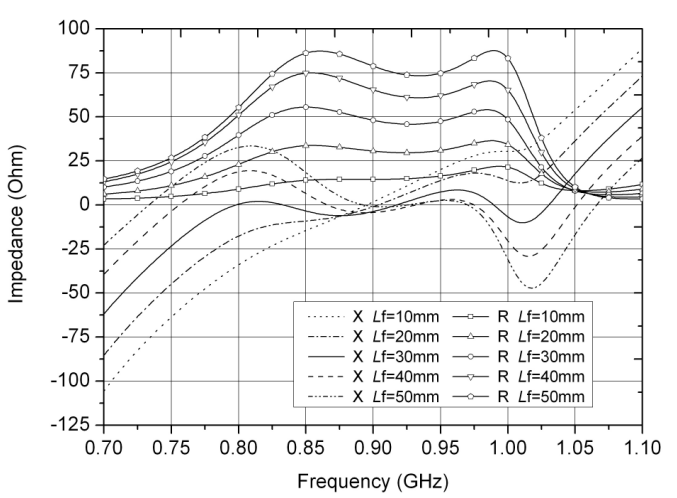

(b)

Fig. 14 Effect of the $L_{\mathrm{f}}$ on the antenna performance. (a) H-plane x-polar at $900 \mathrm{MHz}$. (b) input impedance.

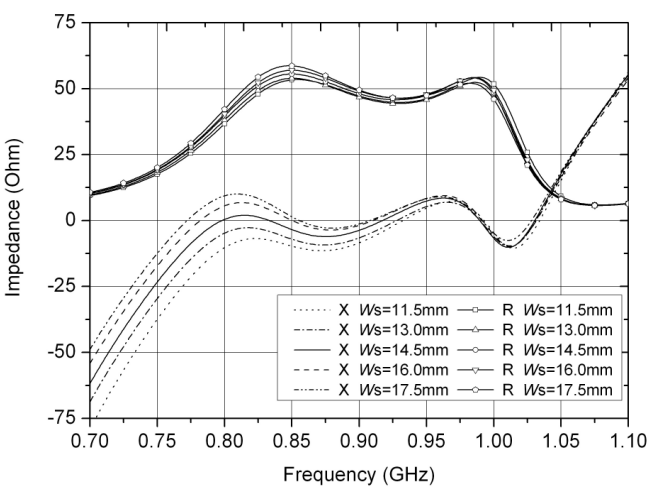

Fig. 15 Effect of the $W_{\mathrm{s}}$ on the input impedance.

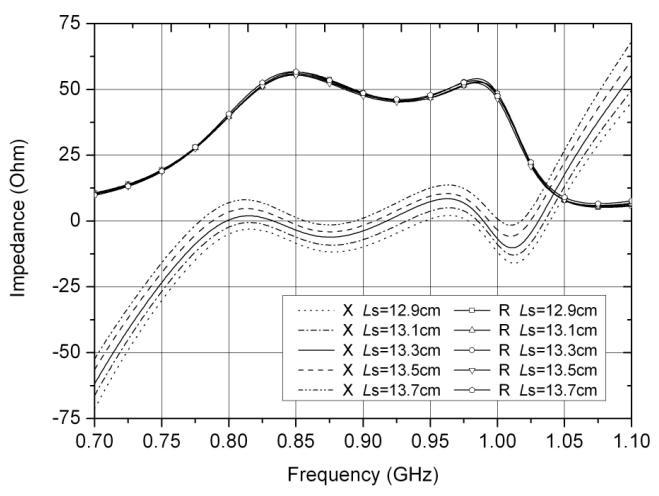

Fig. 16 Effect of the $L_{\mathrm{s}}$ on the input impedance.

\section{Width of Modified L-Strip $\left(W_{s}\right)$}

Fig. 15 exhibits the effect of the width $W_{s}$ of the modified L-strip on the input impedance of the antenna. It is found that a flatter input reactance curve can be obtained by tuning the width $W_{s}$ of modified L-strip, the effect of which on the input resistance is smaller. Similar to $h_{l}$, the crosspolarization levels of the antenna is modestly affected by $W_{s}$ so that the results are not exhibited.

\section{E. Length of Horizontal Segment of Modified L-Strip $\left(L_{s}\right)$}

Contrary to $L_{f}$, the length $L_{s}$ of horizontal segment of the modified L-strip has a greater effect on input reactance of the antenna. As shown in Fig. 16, when changing the length $L_{s}$ from $129 \mathrm{~mm}$ to 137 
$\mathrm{mm}$ the input reactance is uniformly increased, meanwhile the input resistance is hardly affected. The length $L_{s}$ has a greater effect on cross-polarization levels of the antenna, whereas the effect is slight if the length $L_{s}$ is kept within $129-137 \mathrm{~mm}$ so that the results are also not exhibited.

\section{F. Procedures of antenna design}

Based on the foregoing parametric studies, the following procedures are suggested to design the antenna:

1. Calculate the resonant length of the patch $L_{1}$ and $L_{2}$ with $L_{1} \approx 0.40 \lambda_{0}$ and $L_{2} \approx 0.34 \lambda_{0}$.

2. Choose a spacing between the lower patch and the ground $\left(<0.06 \lambda_{0}\right)$ for cross-polarization suppression.

3. Calculate the length of horizontal segment of the modified L-strip $L_{s}$ with $L_{s} \approx 0.40 \lambda_{0}$.

4. Determine the value of $L_{f}$ for which the input resistance at the center frequency $f_{0}$ is $50 \mathrm{Ohm}$.

5. Determine the separation between the patches $h_{l}$ to obtain a flatter input resistance.

6. Determine the value of $W_{s}$ in order to get a flatter input reactance.

7. Tune the value of $L_{s}$ for which the input reactance is near zero.

\section{CONCLUSION}

A wideband dual-layer patch antenna is presented. The proposed antenna has a wide impedance bandwidth of $22 \%(\mathrm{VSWR}<1.2)$ and a low cross polarization level of less than $-20 \mathrm{~dB}$ across the operating bandwidth. The VSWR of the antenna is much lower than other available wideband patch antennas. This is attributed to the dual-layer patch configuration and modified L-strip feed technique. The antenna also has a gain level of about $9 \mathrm{dBi}$. A parametric study and a design guideline of the proposed antenna are also presented.

\section{ACKNOWLEDGMENT}

This work was supported by the National Natural Science Foundation of China (No. 60771032).

\section{REFERENCES}

[1] Y. H. Ge, K. P. Esselle, and T. S. Bird, “A Compact E-shaped Patch Antenna with Corrugated Wings," IEEE Trans. Antennas and Propagation, vol. 54, no. 8, pp. 2411-2413, Aug. 2006.

[2] R. Chair, C. L. Mak, K. F. Lee, K. M. Luk, and A. A. Kishk, "Miniature Wide-Band Half U-Slot and Half E-Shaped Patch Antennas," IEEE Trans. Antennas and Propagation, vol. 53, no. 8, pp. 2645-2652, Aug. 2005.

[3] K. L. Wong, C. L Tang, and J. Y. Chiou, "Broad-band Probe-fed Patch Antenna with a W-shaped Ground Plane," IEEE Trans. Antennas and Propagation, vol. 50, no. 6, pp. 827-831, June 2002.

[4] W. H. Hsu, and K. L. Wong, "Broad-band Probe-fed Patch Antenna with a U-shaped Ground Plane for CrossPolarization Reduction,” IEEE Trans. Antennas and Propagation, vol. 50, no. 3, pp. 352-355, Mar. 2002.

[5] H. Ghannoum, S. Bories, and C. Roblin, "Probe Fed Stacked Patch Antenna for UWB Sectoral Applications," Proc. IEEE International Conference on Ultra-Wideband, pp. 97-102, 2005

[6] M. A. Matin, B. S. Sharif, and C. C. Tsimenidis, "Probe Fed Stacked Patch Antenna for Wideband Applications," IEEE Trans. Antennas and Propagation, vol. 55, no. 8, pp. 2385-2388, Aug. 2007. 
[7] M. T. Islam, M. N. Shakib, N. Misran, and B. Yatim, "Ultrawideband EH Shaped Stack Patch Antenna for Wireless Communications," Proc. IEEE Wireless and Microwave Technology Conference, pp. 1-4, 2009.

[8] Q. Xue, X. Y. Zhang, and C. H. K. Chin, "A Novel Differential-fed Patch Antenna," IEEE Antennas and Wireless Propagation Letters, vol. 5, pp. 471-474, 2006.

[9] Z. N. Chen, and M. Y. W. Chia, "Broad-band suspended probe-fed antenna with low cross-polarization levels," IEEE Trans. Antennas and Propagation, vol. 51, no. 2, pp. 345-346, Feb. 2003.

[10] H.W. Lai, and K.M. Luk, "Design and Study of Wide-Band Patch Antenna Fed by Meandering Probe," IEEE Trans. Antennas and Propagation, vol. 54, no. 2, pp. 564-571, Feb. 2006.

[11] M.K. Meshram, "Analysis of L-strip Proximity Fed Rectangular Microstrip Antenna for Mobile Base Station," Microwave and Optical Technology Letters, vol. 49, no. 8, pp. 1817-1824, Aug. 2007.

[12] W. F. Richards, Y. T. Lo, and D. D. Harrison, “An Improved Theory for Microstrip Antennas and Applications," IEEE Trans. Antennas and Propagation, vol. 29, no. 1, pp. 38-46, Jan. 1981.

[13] Z. N. Chen, and M. Y. W. Chia, "Experimental Study on Radiation Performance of Probe-Fed Suspended Plate Antennas," IEEE Trans. Antennas and Propagation, vol. 51, no. 8, pp. 1964-1971, Aug. 2003. 